\title{
MISZELLE
}

\author{
PETER SEILER
}

\section{Das Lächeln des Cangrande della Scala}

Unter den Skulpturen der Scaliger-Grabmäler in Verona ist die Reiterstatue des Cangrande della Scala (†1329) zweifellos die berühmteste ${ }^{1}$. Die Faszination, die sie immer wieder auslöst, beruht in erster Linie auf ihrer effektvollen illusionistischen Ausrichtung auf den Betrachter: Cangrande ist in Rüstung als paradierender Krieger vergegenwärtigt und zwar so, als habe er gerade sein Schlachtroß angehalten, um sich seinen Untertanen zuzuwenden ${ }^{2}$. Das Erstaunliche dabei ist sein Lächeln (Abb. I $)^{3}$. Wie ist dieser bei mittelalterlichen Herrscherbildnissen ansonsten nicht anzutreffende Gesichtsausdruck zu verstehen? Da der Dargestellte durch den narrativen Reliefzyklus des Sarkophags als Eroberer der Trevisaner Mark kommemoriert wird, liegt es nahe, sein Lächeln als triumphierendes Siegerlächeln aufzu-

I Zu den Grabmälern der Scaliger siehe Peter Seiler, Mittelalterliche Reitermonumente. Studien zu personalen Monumentsetzungen in den italienischen Kommunen und Signorien des I3. und 14. Jabrbunderts, Phil. Diss., Universität Heidelberg 1989, Bd. I, 260319 und Bd. II, 97-268; Ders., La trasformazione gotica della magnificenza signorile: committenza viscontea e scaligera nei monumenti sepolcrali dal tardo duecento alla metà del trecento, in: Presenze del Gotico Europeo in Italia, hrsg. von Martina Bagnoli und Valentino Pace, Neapel I994, I I9- I40, bes. I32-I34 und Ders., Residenz, Kirche, Grablege - Zur Entstehungsgeschichte des Residenzensembles der Scaliger in Verona, in: Architectural Studies in Memory of Richard Krautheimer, hrsg. von Cecil L. Striker, Mainz I 996, I5 I-I56.

2 Die sicherlich erst in den vierziger Jahren des I4. Jahrhunderts entstandene Reiterstatue wurde I9I0 durch eine Kopie ersetzt. Das Original befindet sich im Museo di Castelvecchio in Verona. Vgl. hierzu Gian Maria Varanini, Cipolla, Simeoni, Da Lisca: la corrispondenza sulla statua equestre di Cangrande I, in: $L a$ statua equestre di Cangrande I della Scala. Studi, ricerche, restauro, a cura di Sergio Marinelli e Giulia Tamanti, Verona 1995, 5i-59. Zum Erhaltungszustand der Statue siehe Giulia Tamanti, Le vicende conservative della statua equestre di Cangrande I del- fassen ${ }^{4}$. Das Gesicht der Liegefigur, die den toten Helden zeigt, stimmt jedoch mit dem der Reiterstatue motivisch überein (Abb. 2). Die heitere Miene kann demzufolge nicht (oder zumindest nicht ausschließlich) als mimischer Ausdruck einer momentanen Gefühlslage verstanden werden. Sie diente offenbar zur Charakterisierung der Persönlichkeit des Scaligers.

War Cangrande ein heiterer Mensch? Beruhte sein Charisma nicht nur auf kriegerischen Erfolgen, sondern auch auf persönlichem Frohsinn? Hat der Bildhauer versucht, die charmante aria des Scaligers zu verewigen?s Man hat tatsächlich die Auffassung vertreten, es handele sich um ein »ritratto dal vivo«, das Cangrande so darstelle, wie er aussah und wie er war'. Als man I92 I den Sarkophag öffnete, glaubte man, die Physiogno-

la Scala, in: ibid., 64-94 und die in demselben Band publizierten Ergebnisse technischer Untersuchungen. Zur Entstehungsgeschichte des Grabmals von Cangrande siehe Peter Seiler, Per un'identificazione del sarcofago a rilievo del Sepolcreto scaligero di Verona, in: Bisanzio e l'Occidente: arte, archeologia, storia. Studi in onore di Fernanda de' Maffei, Rom 1996, 54I-555 und ders., Untersuchungen zur Entstebungsgeschichte des Grabmals von Cangrande I. della Scala (im Druck).

3 Zum Lächeln des Cangrande gibt es zahlreiche kommentierende Bemerkungen, Mutmaßungen und Deutungsversuche, auf die ich hier nicht im Einzelnen eingehen kann. Auf die wichtigsten Forschungsmeinungen wird in den folgenden Anmerkungen hingewiesen.

${ }_{4} \mathrm{Zu}$ den verschiedenen Anlässen und Varianten des Lächelns bzw. Lachens in kriegerischen Kontexten (Demonstration von Unerschrockenheit und Überlegenheit vor dem Kampf, Frohlocken über besiegte Feinde) vgl. die der antiken und mittelalterlichen Epik entnommenen Belege in Karl Richard Kremer, Das Lachen in der deutschen Sprache und Literatur des Mittelalters, Phil. Diss., Bonn 196r, 54, 6I, I29, I44, I5 fff., I75, I77. Zum "Gefahrverlachen « und zum »erlösenden Lachen« nach einer Gefahr vgl. auch Martin J. Schubert, Zur Theorie des Gebarens 


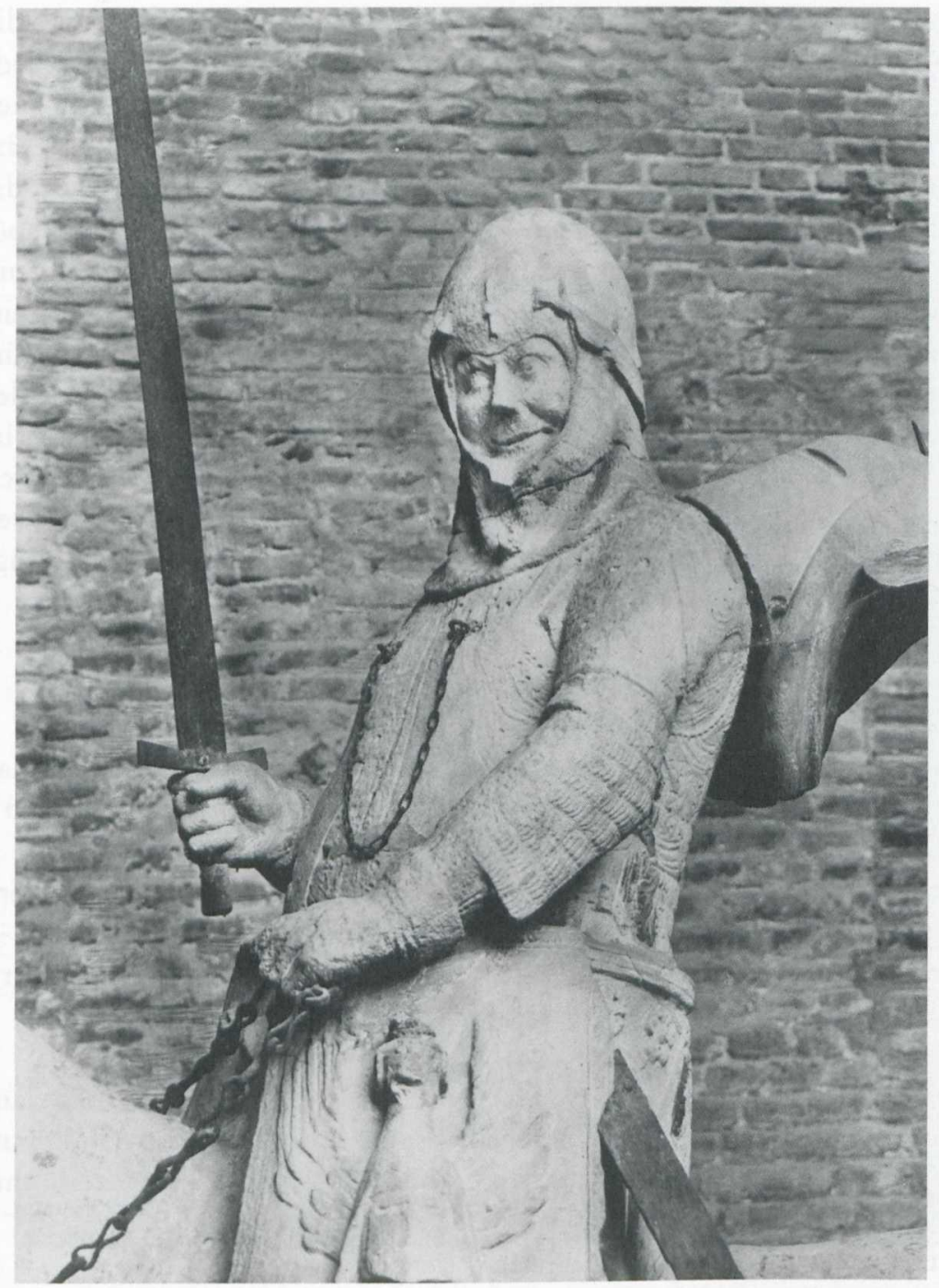

I. Grabmal des Cangrande della Scala, Verona, Reiterstatue, Detail

im Mittelalter. Analyse von nichtsprachlicher Äußerung in mittelhochdeutscher Epik. Rolandslied, Eneasroman, Tristan (Kölner Germanistische Studien, Bd. 31), Köln-Wien 1991, 92.

5 Der Begriff "aria" war nach dem Zeugnis Petrarcas bei zeitgenössischen Malern in Gebrauch. Er soll von diesen zur Kennzeichnung der für eine Person besonders charakteristischen Gesichtszüge verwandt worden sein. Francesco Petrarca, Familiarum rerum, Lib. XXIII, 19, 78 -94. Le Familiari, Edizione critica a $\mathrm{Cu}$ ra di Vittorio Rossi, vol. IV per cura di Umberto Bosco, Libri XX-XXIV, Florenz I942, 206; vgl. hierzu auch Ernst H. Gombrich, Maske und Gesicht. Die Wahrnehmung physiognomischer Ähnlichkeit im Leben und in der Kunst, in: Ernst H. Gombrich, Julian
Hochberg, Max Black, Kunst, Wabrnehmung, Wirklichkeit, Frankfurt/Main 1972, I7.

6 Carlo Cipolla, La statua equestre di Cangrande I a Verona, in: Arte e Storia, ser. IV, 29, 1910, 225-228; Fernanda De Maffei, Le arche scaligere di Verona, Verona 1955, 52 und 54. Bettini versuchte mit der angenommenen Portraitähnlichkeit sogar die Frühdatierung der Reiterstatue "um I330 zu begründen, siehe Sergio Bettini, La pittura gotica internazionale a Verona. Appunti dalle lezioni di Storia dell'Arte Medioevale, Istituto di Storia dell'arte - Università di Padova, Anno Accademico $1973-74$, I 8: »(Mi) sembra incontestabile che (il Cangrande a cavallo) sia un autentico ritratto del sran lombardos, e un ritratto eseguito da vivo. È difficile, quasi impossibile supporre che un artista, sia pure 
mie des Bildnisses in derjenigen des Leichnams wiederzuerkennen (Abb.3)7. In einem damals abgefaßten Bericht ist sogar vom Lächeln des Scaligers die Rede: „Cangrande (...), conserva tutta la sua pelle che mostra la sua forte musculatura; i suoi capelli ricciuti sono biondi castagni, la sua fronte è lisca e spaziosa, gli zigomi porgono un poco, il volto è regolare e rotondo, gli occhi guardano quasi con un'espressione di vita; egli sembra ancora sorridere dalla sua bocca semiaperta che mostra i denti conservatissima $\ll^{8}$.

Ende der zoer Jahre äußerte Harald Keller eine abweichende Meinung und er registrierte andere Übereinstimmungen: »Natürlich zeigte das Gesicht nicht das archaische Lächeln der Reiterfigur, aber die kleine Nase mit den Flügeln, die etwas nach oben gezogen sind und die knappen Lippen waren ihm wirklich eigen - und gerade das sind ja die Eigentümlichkeiten der Skulptur. Auch der Schädel des Toten war so abnorm steil wie der des Standbildes «?

Versucht man die Angaben zu den einzelnen physiognomischen Details zu überprüfen und berücksichtigt man dabei auch die Liegefigur (was z.B. Keller nicht tat), dann stellen sich Irritationen ein: Waren Kopf und Gesicht des Scaligers eher rund oder »steil«? Wie sah seine Nase aus? War sie »lievemente aquilino ${ }^{10}$ oder gerade, wie die beider Bildnisse? Hatte Cangrande tatsächlich Augenbrauenbögen, die so rund und hoch in die Stirn ragten wie diejenigen modisch geschminkter Madonnen der transalpinen Gotik? Kann man wirklich davon ausgehen, daß der lebende Cangrande ebenso schmale Lippen hatte wie sein ausgetrockneter Leichnam oder ist die Übereinstimmung von Bildnissen und Leichnam hinsichtlich dieses Merkmals zufällig? Zu sicheren Antworten auf diese Fragen wird man kaum gelangen können. Aber sind die physiognomischen Details überhaupt von Bedeutung? Könnte es nicht sein, daß der Bildhauer sich gar nicht darum bemühte, diese ganz präzise wiederzugeben, da es ihm in erster Linie darauf ankam, die individuelle aria des Cangrande darzustellen? Man zögerte immer wieder, den Glauben an die personale Authentizität der Heiterkeit des Scaligers in Frage zu stellen, da es für diesen Charakterzug einen schriftlichen Quellenbeleg zu geben scheint: die in dem Serventese in morte di Cangrande enthaltene Personenbeschreibung. In ihr heißt es, Cangrandes Gesicht sei "pieno di allegrezza« gewesen. Die Bedeutung dieses Zeugnisses erschließt sich jedoch erst aus dem inhaltlichen Zusammenhang:

El nobele barun ingraciato

Misser Can de la Scala era cla(mat)o;

Per tutto lo mondo era disidrato De vedere.

De soe prodeçe asai sen poria dire

Plu che de barun che may sia;

Per tutto 'I mundo l'à fatto florire La soa posana.

Misser Can de la Scala, franca lança (El plu le) al che sia de qui a França (per) lo mondo ello porta nomennaça De prodeçe.

Franco barone e de gran çentileçe, Largo e cortexe e nobil per çerteçe, El so viso era pleno d'alegreça One staxone.

Misser Can de la Scala, quel barone, In la Vergene avea gran devocione; Fiolo de Deo, che soferi pasione, O li perdona ${ }^{11}$. di eccezionali virtù interpretative ed evocative, e magari con l'ausilio di qualche ritratto in pittura o miniatura (oggi tuttavia inesistente) abbia potuto conferire, a distanza di almeno vent'anni dalla morte del principe, e presumibilmente senza averlo mai veduto vivente, una caratterizzazione così incisamente icastica non solo dei suoi lineamenti, ma dei suoi gesti del suo sumore< perculiari: giacchè questo è appunto il dato più vistoso di un così eccezionale ritratto. « Vgl. auch Gian Lorenzo Mellini, Tracce di Giotto a Verona, in: Critica d'arte 8, 1961, 17-26 und ders., Scultori veronesi del Trecento, Mailand o.J. (1971), 94-100, bes. 96.

7 Siehe Augusto Ferrero, Ciò che resta di Can Grande Signore di Verona, in: Rassegna d'arte 22, I922, 139.

8 »Verona - Arca di Cangrande: - Ricognizione delle Spoglie«, Verona, Archiv der Soprintendenza (unsig- 


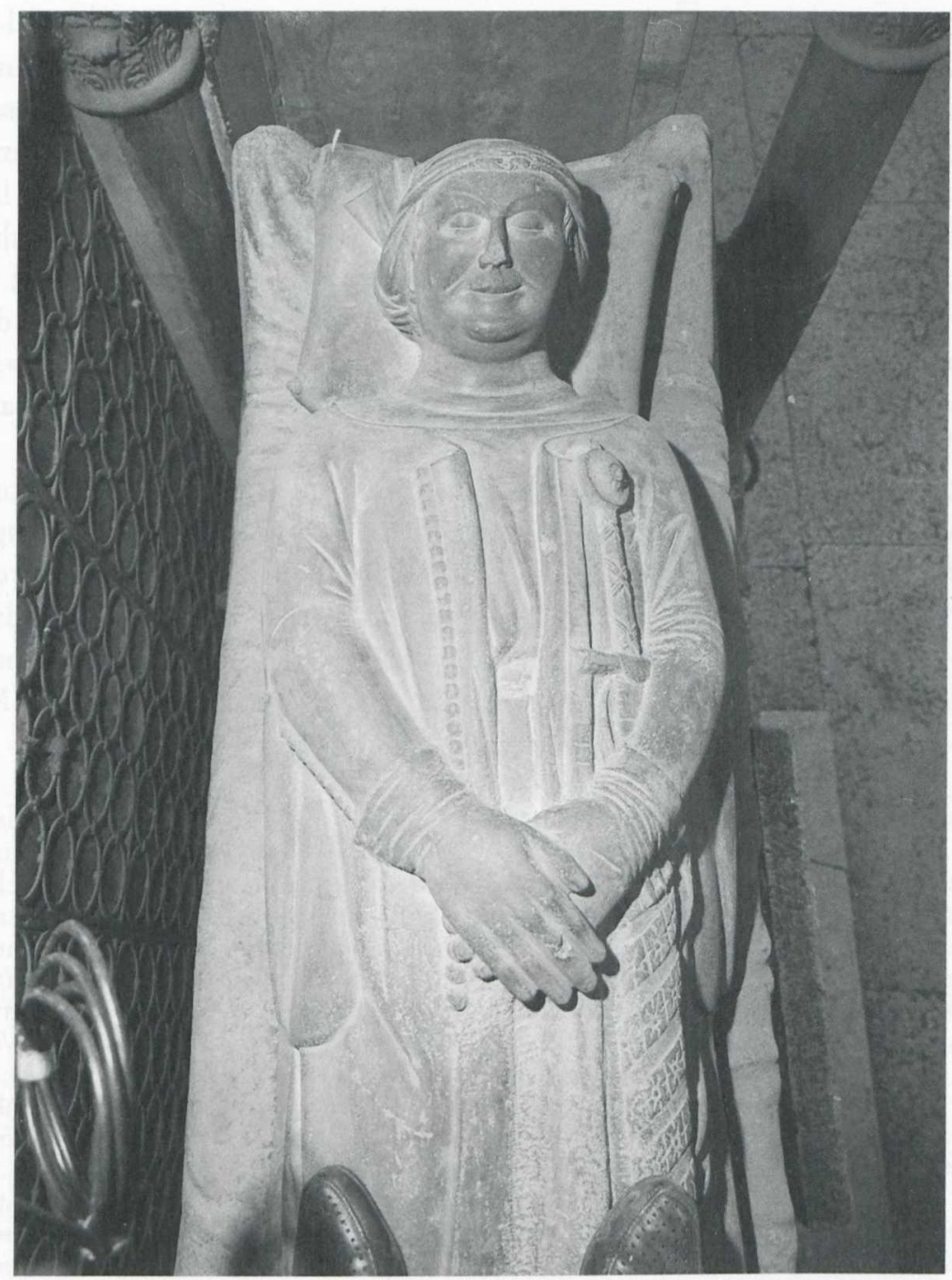

2. Grabmal des Cangrande della Scala, Verona, Liegefigur, Detail

Es verhält sich mit dieser Beschreibung ebenso wie mit vielen panegyrischen Herrscherdeskriptionen. Der Dichter bemühte sich nicht darum, Cangrande zu schildern, wie er wirklich war,

nierte maschinenschriftliche Kopie des Originals).

9 Harald Keller, Die Entstehung des Bildnisses am Ende des Hochmittelalters, in: Römisches Jahrbuch für Kunstgeschichte 3, 1939, 330. Keller stützte sich auf die Ausführungen von Ferrero (wie Anm. 7) und auf eine von diesem publizierte Zeichnung, die Angelo Dall'Oca Bianca I92 I während der Ôffnung des Sarkophags angefertigt hatte. sondern er stellte ihn als ideale Verkörperung des guten Herrschers dar. Die Charakterisierung konzentriert sich auf Persönlichkeitsmerkmale, die zum traditionellen Katalog der Eigenschaften

Io Antonio Avena, La salma e la tomba di Cangrande I della Scala, in: Dante e Verona. Studi pubblicati a cura di Antonio Avena e Pieralvise Alighieri in occasione del seicentenario dantesco, Verona 1921, 402.

I I Carlo Cipolla/Flaminio Pellegrini, Poesie minori riguardanti gli Scaligeri, in: Bolletino dell'Istituto Storico Italiano 24, 1902, 59 Nr. XXVI; vgl. die Hinweise in Avena (wie Anm. 10), 417-4I8 
vorbildlicher Herrscher gehörten, wie vornehme Geburt, Schönheit, Tapferkeit, Ruhm, Macht, Freigiebigkeit, höfische Manieren und Frömmigkeit. Zwar findet man auch in konventionellen mittelalterlichen Herrscherbeschreibungen gelegentlich Ansätze individueller Charakterisierung, aber die heitere Miene des Cangrande ist nicht als individueller Wesenszug aufzufassen. Sie ist ein traditionelles Attribut des tugendhaften Herrschers. So wie zum Beispiel in der Antike Sueton in seiner Vita des Augustus dessen heiteren Gesichtsausdruck hervorhob ${ }^{12}$, so haben auch im Mittelalter Dichter und Geschichtsschreiber die hilaritas von Herrschern betont und ihnen ein frohes und freundliches Gesicht zugeschrieben $^{13}$. Gelegentlich ist hierbei auch von einer besonderen Neigung zum Lächeln die

I 2 Sueton, Augustus c. 79, r: "vultu erat vel in sermone vel tacitus adeo tranquillo serenoque«, zit. nach C. Suetonius Tranquillus, Die Kaiserviten / De vita Caesarum - Berübmte Männer / De viris illustribus, Lat.dt., hrsg. und übers. von Hans Martinet, Düsseldorf/Zürich, 274. Die heitere Miene des Königs ist auch im Alten Testament belegt: Prv I6, Is »in hilaritate vultus regis vita et clementia eius quasi imber serotinus", und I9,I2 "sicut fremitus leonis ita et regis ira et sicut ros super herbam ita hilaritas eius «, zit. nach Biblia Sacra iuxta vulgatam versionem ..., hrsg. von Bonifatius Fischer und Robert Weber, Stuttgart ${ }^{3} \mathrm{I} 984$.

I 3 G. Pedrino, Cronica del suo tempo, Edita da G. Borghezio e M. Vattasso, con note storiche di A. Pasini, II, Rom 1934, 440 (zu Sinibaldo Ordelaffi, Signore von Forli, gest. I386): »homo molto aliegro«; zu Azzo Visconti siehe Pietro Azario, Liber gestorum in Lombardia, RIS XVI, 3 I 4: "Qui quamquam juvenis, senex fuit in bonitate $\&$ in virtute, laetus facie, crinibus candidus, gracilis corpore, sed pinguedine intermixtus, affabilis, tractabilis, benignus «; Bernardo Corio, Storia di Milano (1503), a cura di A. Morisi Guerra I, Turin 1978, 746: »Fu Azo de commune statura, rotondo di faccia et alegro «; in einem von Francesco Cognasso, I Visconti, Varese 1966, 279 zitierten Gedicht über Bernabò Visconti heißt es: »Che cosi ride e sicuro esser crede / Quelgli è il milanese Barnabò «. Zum Vorkommen von "hilari/hilari vultu « in spezifischen Handlungszusammenhängen siehe Fernand Vercauteren, Avec le sourire ..., in: Mélanges offerts à Rita Lejeune, Bd. I, Gembloux 1969, 45-s6 (anläßlich von Schenkungen); Hatto Kaffelz, Das Standesethos des Adels im IO. und II. Jahrhundert, Phil. Diss, Würzburg I96I, 68ff. (bei Gastmälern). Vgl. auch Heinrich Fichtenau, Lebensordnungen des 10. Jahr-
Rede. So erwähnt zum Beispiel im I2. Jahrhundert Acerbo Morena nicht nur das heitere Gesicht des Friedrich Barbarossa, sondern weist zusätzlich noch darauf hin, man hätte den Eindruck gehabt, als ob er immer lächeln wolle (ut semper velle ridere $)^{14}$. Ein ähnliches mimisches Gebaren soll im I4. Jahrhundert nach dem Zeugnis des Anonimo Romano auch dem Römer Cola di Rienzo eigen gewesen sein: »Era bello omo e in soa vocca sempre riso appareva in qualche muodo fantastico ${ }^{15}$.

Hat man diese, durch traditionelle Herrscherbeschreibungen und höfische Tugendkataloge geprägten Zeugnisse vor Augen, dann wird deutlich, daß Mutmaßungen über die charakterliche Authentizität der heiteren Miene des Cangrande von zweifelhaftem Wert sind ${ }^{16}$. Man muß jedoch

bunderts. Studien über Denkart und Existenz im einstigen Karolingerreich, I992, 53 f. und 88f.; zu "hilaritas « und »iocunditas als höfische Tugenden siehe Paul Gerhard Schmidt, Curia und curialitas. Wort und Bedeutung im Spiegel der lateinischen Quellen, in: Curialitas. Studien zu Grundfragen der höfischritterlichen Kultur, hrsg. von Josef Fleckenstein, Göttingen 1990, 17; Sabine Krüger, »Verhöflichter Ritter « und miles illiteratus, in: ibid., 339; Thomas Zotz, Urbanitas. Zur Bedeutung und Funktion einer antiken Wertvorstellung innerhalb der höfischen Kultur des hohen Mittelalters, in: ibid., 395, 402f., 405. Zu den vielfältigen Einstellungen zu Heiterkeit und Lachen im Mittelalter siehe auch Ernst Robert Curtius, Europäische Literatur und lateinisches Mittelalter, Bern und München ${ }^{9}$ I978, 419-434, Exkurs IV. Scherz und Ernst in mittelalterlicher Literatur; Kremer, Das Lachen in deutscher Sprache und Literatur des Mittelalters (wie in Anm. 4); Philippe Ménard, Le rire et le sourire dans le roman Courtois en France au Moyen Age (1150-1250), Genève 1969; Gerhard Schmitz, ... quod rident homines, plorandum est. Der ,Unwert des Lachens in monastisch geprägten Vorstellungen der Spätantike und des Mittelalters, in: Franz Quarthal/Winfried Setzler (Hrsg.), Stadtverfassung - Verfassungsstaat - Pressepolitik. Festschrift für Eberhard Naujoks, Sigmaringen 1980, 3-I 5; Giovanni Ciappelli, Ridere nel Medioevo, in: Quaderni medievali 21, I989, I20-I27; Schubert (wie Anm.4), bes. 89-93; Jacques Le Goff, Laughter in the Middle Ages, in: J. Bremmer und H. Roodenburg (Hrsg.), A Cultural History of Humour from Antiquity to the Present Day, Cambridge 1997, 40-53. Zum Problemfeld »Lachen und Karneval« vgl. bes. Dietz-Rüdiger Moser, Lachkultur des Mittelalters? Michael Bachtin und die Folgen seiner Theorie, in: Euphorion. Zeit- 


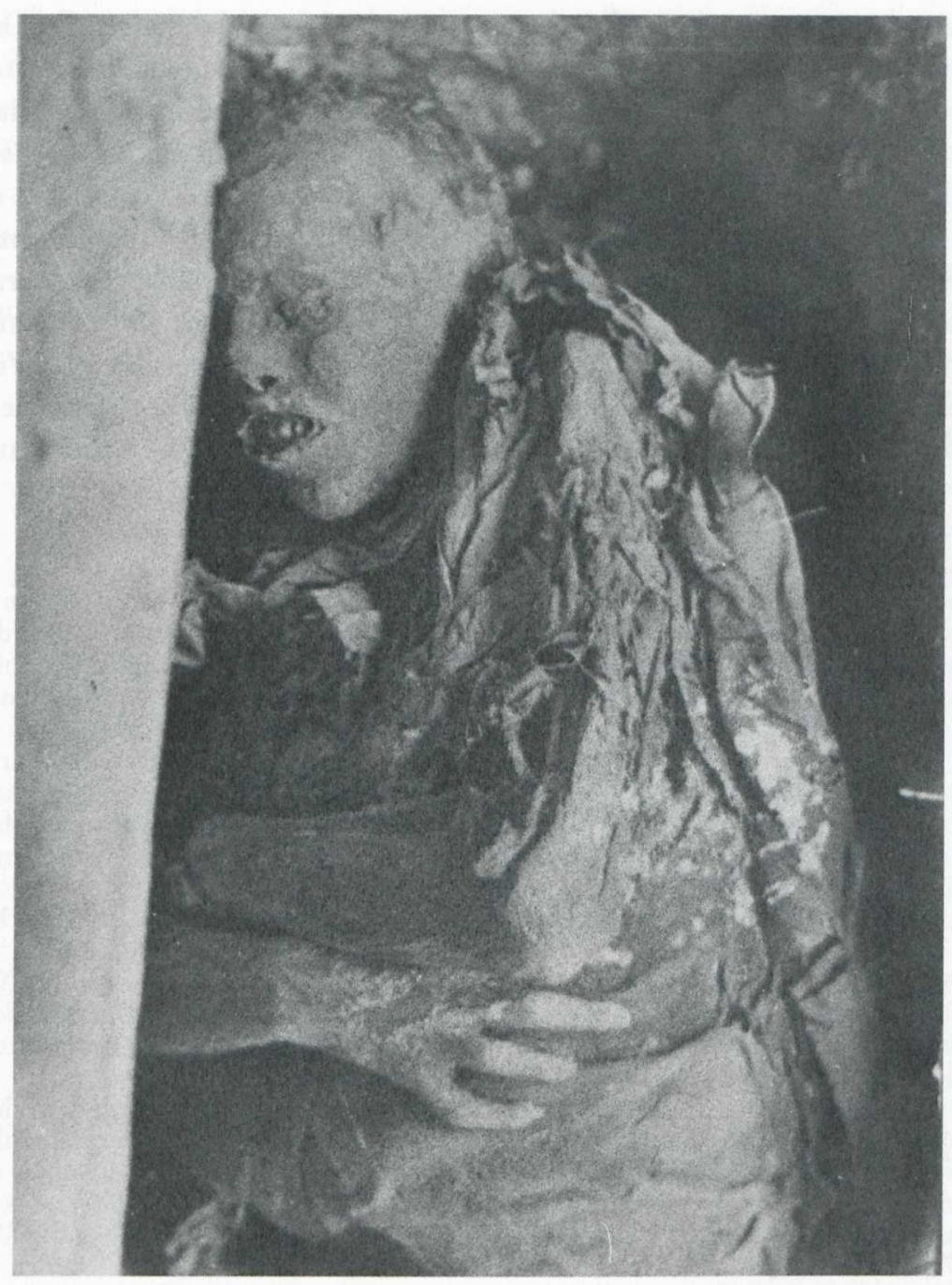

3. Grabmal des Cangrande della Scala, Sarkophag mit dem mumifizierten Leichnam

schrift für Literaturgeschichte 84, I990, 89-III und die mit diesem Aufsatz zusammenhängenden Diskussionsbeiträge von Elena Nährlich-Slateva, Aaron J. Gurjewitsch, Dietz-Rüdiger Moser und Heidy Greco-Kaufmann in den beiden folgenden Jahrgängen dieser Zeitschrift.

I4 Annales Laudenses auctoritate Ottone et Acerbo Morenis a. II $33-1168$, hrsg. von Philipp Jaffé, MGH SS XVIII, 640; vgl. hierzu Herbert Grundmann, Der Cappenberger Barbarossakopf und die Anfänge des Stiftes Cappenberg, Köln/Graz I959, s Iff.; Zotz (wie Anm. I3), 395, 403. Die Formel »semper facie hilaris" kennt auch Donizio, der Verfasser des panegyrischen Gedichts auf die Markgräfin Mathilde, siehe Vita Mathildis celeberrimae principis Italiae. Carmine scripta a Donizio Presbytero qui in arce Canusina vixit, RIS n. Ed. V/2, hrsg. von Luigi Simeoni, Bologna 1940,56 und II 8 .

Is Anonino Romano, Chronica, Edizione critica a cura di Giuseppe Porta, Milano 1979, I43.

I6 Es ist aufgrund der zitierten Belege meines Erachtens nicht angebracht, anhand vager Anhaltspunkte das Lächeln des Cangrande auf orientalische Einflüsse zurückführen zu wollen. Antonio Scolari hat Cangrande eine auf seinem Namen basierende, ausgeklügelte herrscherliche Selbstinszenierung unterstellt. Der Veroneser Signore habe, inspiriert durch Berichte über den Gran Kan dei Tartari, gezielt versucht, sich ein orientalisches Herrscherimage zu verschaffen. In Zusammenhang mit diesem Bestreben verwies Scolari 
nicht notwendigerweise dem Lächeln des Scaligers jeglichen historischen Realitätsgehalt absprechen. Es mag durchaus mehr als eine literarische Fiktion gewesen sein. Möglicherweise hat Cangrande tatsächlich in der Öffentlichkeit häufig demonstrativ gelächelt. Das öffentliche zur Schau tragen von Heiterkeit und Freundlichkeit gehörte zu dem ritualisierten Gebaren, das man von tugendhaften Herrschern wie auch von anderen Personen bei Hof erwartete. "In publico hylaritas « lautet die zweite Verhaltensnorm einer

ohne nähere Begründung auch auf die Heiterkeit des Scaligers: "C'era nel costume di Can Grande, quale Dante lo vide, una calcolata ricerca di esotismo orientale, che si confaceva col suo volto dall'aperto sorriso." Antonio Scolari, Verona e gli Scaligeri nella vita di Dante, in: Kat. Dante e Verona, Verona 1965, XXI. Vgl. auch ders., Il messia dantesco, Bologna I9I3, 3 Iff., 39, $40 \mathrm{Anm}$. I, 5sff., Iogff. Scolaris suggestive Mutmaßungen fungierten als Inspirationsquelle für eine Reihe weiterer Beiträge, in denen über orientalische Einflüsse am Hof der Scaliger spekuliert wird, vgl. z.B. Gian Lorenzo Mellini, Verona, la Corte sveva, l'Orienta e le origini del Gotico, in: Labyrinthos 9, 1986, 3-49; Giorgio Battistoni, Dante, l'Islam e altre considerazioni, in: Labyrinthos I I 1987, 26-65, bes. 36 und 49 ff.; ders., Simboli e mitografie intorno a Can Grande della Scala I und 2, in: Labyrinthos I3/ I6, $1988 / 89,35-62$, bes. 62 und $19 / 20$, I991, 59-103. Den Vertretern der Orientthese ist bisher entgangen, daß tatsächlich eine auf orientalischer Kunst basierende Darstellung eines lächelnden Gran Khan überliefert ist. Es handelt sich um eine Miniatur, die in einer aus der zweiten Hälfte des I 4. Jahrhunderts stammenden Genueser Handschrift enthalten ist (London, British Library: ms. Add. 27695, fol. 13r). Die Darstellung zeigt den Gran Khan »alla turca« sitzend während eines Banketts. Sie dient freilich nicht der Vergegenwärtigung eines positiven Herrschervorbilds, sondern der Veranschaulichung des Vizio della gola«. Siehe Wolfram Prinz, I Tartari nella Pittura Italiana del Trecento, in: Studi di Storia dell'Arte sul Medioevo e il Rinasciemento nel Centenario della Nasciatà di Mario Salmi (= Atti del Convegno Internazionale Arezzo-Firenze, 16-19 Novembre 1989), Firenze 1993, vol.I., 4I8, fig. 8. Zum Herrscherimage des Cangrande vgl. zuletzt Andrea di Salvo, "Celebrazioni politiche d'occasione«: Il Caso dei primi Scaligeri, in: Le forme della propaganda politica nel due e nel trecento, hrsg. von Paolo Cammarosano (Collection de l'Ecole Française de Rome. 20I), Rom 1994, 287-310 und Gian Maria Varanini, Propaganda dei regimi signorili: Le esperienze venete del Trecento, in: ibid., $31 \mathrm{I}-343$, bes. $316 \mathrm{ff}$.

17 Schmidt (wie Anm. I3), 17. Bei welchen Anlässen das spätmittelalterlichen Liste von septem curialitates $^{17}$.

Mit dem Nachweis, daß das literarisch bezeugte heitere Gesicht des Cangrande eine konventionelle Maske war, ist das Rätsel der beiden lächelnden Bildnisse des Signoren freilich noch nicht vollständig gelöst. Schließlich war es im Bereich der mittelalterlichen Bildniskunst nicht üblich, weltliche (oder geistliche) Würdenträger mit heiteren Gesichtszügen darzustellen ${ }^{18}$. Sicherlich muß man auch die Ausbreitung des "gothic

zur Schau tragen einer heiteren Miene zum ritualisierten Gebaren von Herrschern gehörte, ist bisher nur ansatzweise erforscht. Es wurde auf Schenkungen und Gastmälern verwiesen (vgl. hierzu Anm. I3). Zu berücksichtigen sind auch Gesandtschaftsempfänge. Der die Eroberung Trevisos und den Tod des Cangrande schildernde lateinische Carmen berichtet zum Beispiel, der Veroneser Signore habe eine Trevisaner Gesandtschaft »hilari vultu « empfangen. Siehe Antonio Medin, La resa di Treviso e la morte di Can Grande I della Scala: cantare del secolo XIV, in: Archivio Veneto, ser. 2, 3I, I886, 374. Zum Bereich der demonstrativ-rituellen Kommunikation in mittelalterlicher Öffentlichkeit vgl. Gerd Althoff, Spielregeln der Politik im Mittelalter. Kommunikation in Frieden und Fehde, Darmstadt 1997, bes. 29, 206, 223, 279 (zu hilaritas und tristitia).

I 8 Im Zusammenhang mit dem Lächeln des Cangrande wurde vielfach auf die Statue des hl. Zeno in San Zeno in Verona hingewiesen, die unter der volkstümlichen Bezeichnung "S.Zeno che ride «ekannt ist. Das Lächeln ist bei dieser Thronfigur jedoch in anderer Weise dargestellt, so daß letztlich ungewiß bleibt, ob irgendein Zusammenhang mit dem Lächeln des Cangrande bestand. Das Lächeln des hl. Zeno dürfte in der »hilaritas heiliger Bischöfe eine Erklärung finden. Vgl. hierzu die von Vercauteren (wie Anm. I3), I6f. zitierten hagiographischen Quellen. Von Interesse ist in diesem Zusammenhang auch das sanfte Lächeln der Liegefigur des Grabmals von Giovanni D'Aragona (Tarragona, Cattedrale). Giovani d'Aragona, der Erzbischof von Tarragona war, starb I 334 "in odore di santità . Siehe Giovanni Previtali, Il sepolcro di Giovanni d'Aragona: un suggerimento, in: ders., Studi sulla scultura gotica in Italia, Turin I991, 92-99.

I9 Siehe hierzu Seiler, La traformazione gotica (wie Anm. I), I32. Zur Verbreitung des sog. gotischen Lächelns vgl. Jan Svanverg, The Gothic Smile, in: Künstlerischer Austausch - Artistic Change. Akten des XXVIII. Internationalen Kongresses für Kunstgeschichte, Berlin, 15.-20. Juli 1992, hrsg. von Thomas W. Gaehtgens, Bd.2, 357-370; Paul Binski, The Angel Choir at Lindcoln and the Poetics of the Gothic Smile, in: Art History 20/3, 1997, 350-374. 
smile « und die Gotikrezeption des sogenannten Maestro delle Arche Scaligere als Voraussetzungen einkalkulieren ${ }^{19}$. Aber auch dann bleiben noch Fragen offen: Warum hat der Bildhauer gerade Cangrande mit lächelnder Miene verewigt? Warum nicht auch dessen Nachfolger Mastino? Hat er auf Anweisung gehandelt oder aus eigenem Antrieb? Wollte er womöglich eine persönliche Sympathie für Cangrande zum Ausdruck bringen? Wie es auch immer gewesen sein mag, willentlich oder nichtwillentlich hat er durch die heitere Miene des Scaligers nicht unwesentlich dazu beigetragen, daß dieser berühmter wurde und mehr Sympathien auf sich zog als sein ehrgeiziger Neffe Mastino. Vom Charme des steinernen Cangrande betört, hat man - alle Zweifel in den Wind schlagend - nicht gezögert, dessen physiognomische Maske für ein Gesicht zu halten. 